\title{
Patterns of Bidirectional Communication between Cortex and Basal Ganglia during Movement in Patients with Parkinson Disease
}

\author{
Elodie Lalo, ${ }^{1}$ Stéphane Thobois, ${ }^{2}$ Andrew Sharott, ${ }^{4}$ Gustavo Polo, ${ }^{3}$ Patrick Mertens, ${ }^{3}$ Alek Pogosyan, ${ }^{5}$ and Peter Brown ${ }^{5}$ \\ ${ }^{1}$ Laboratory of Motor Activity and Ergonomics, University of Orléans, 45067 Orléans Cedex 2, France, ${ }^{2}$ Department of Neurology C, Inserm, U864, and \\ ${ }^{3}$ Department of Neurosurgery, University Lyon I, Hospital for Neurology Pierre Wertheimer, 69003 Lyon, France, ${ }^{4}$ Department of Neurophysiology and \\ Pathophysiology, University Medical Center Hamburg-Eppendorf, 20246 Hamburg, Germany, and ${ }^{5}$ Sobell Department of Motor Neuroscience and \\ Movement Disorders, Institute of Neurology, London WCIN 3BG, United Kingdom
}

Cortico-basal ganglia networks are considered to comprise several parallel and mostly segregated loops, where segregation is achieved in space through topographic connectivity. Recently, it has been suggested that functional segregation may also be achieved in the frequency domain, by selective coupling of related activities at different frequencies. So far, however, any coupling across frequency in the human has only been modeled in terms of unidirectional influences, a misplaced assumption given the looped architecture of the basal ganglia, and has been considered in static terms. Here, we investigate the pattern of bidirectional coupling between mesial and lateral cortical areas and the subthalamic nucleus (STN) at rest and during movement, with and without pharmacological dopaminergic input, in patients with Parkinson's disease. We simultaneously recorded scalp electroencephalographic activity and local field potentials from depth electrodes and deduced patterns of directed coherence between cortical and STN levels across three frequency bands [sub- $\beta$ (3-13 $\mathrm{Hz}), \beta(14-35 \mathrm{~Hz}), \gamma(65-90 \mathrm{~Hz})$ ] in the different states. Our results show (1) asymmetric bidirectional coupling between STN and both mesial and lateral cortical areas with greater drives from cortex to STN at frequencies $<35 \mathrm{~Hz},(2)$ a drop of $\beta$ band coupling driven from mesial cortex to STN during movement, and (3) an increase in symmetrical bidirectional drives between STN and mesial cortex and in lateral cortical drive to STN in the $\gamma$ band after dopaminergic therapy. The results confirm a bidirectional pattern of cortico-basal ganglia communication that is differentially patterned across frequency bands and changes with movement and dopaminergic input.

Key words: directed transfer function; cortex; subthalamic nucleus; basal ganglia; Parkinson's disease; oscillations

\section{Introduction}

How subcortical and cortical motor areas interact from moment to moment in humans is poorly understood. The current use of stimulation through deep-brain electrodes to treat movement disorders such as Parkinson's disease (PD) provides a remarkable opportunity to investigate subcortico-cortical interactions at high temporal resolution by simultaneously recording cortical electroencephalographic (EEG) and basal ganglia (BG) local field potential (LFP) activities. BG LFPs, just as the EEG LFPs, provide an index of synchronized subthreshold and suprathreshold activities in local neuronal populations (Hammond et al., 2007).

Using this approach, coupling of activities has been demonstrated in terms of coherence between cortical EEG and BG LFPs in patients at rest (Williams et al., 2002; Fogelson et al., 2006). These studies suggested a differential coupling of BG activities at

Received Aug. 28, 2007; revised Jan. 10, 2008; accepted Jan. 18, 2008.

This work was supported by the Medical Research Council of the United Kingdom (P.B.), the France Parkinson Association (E.L.), and the Bundesministerium für Bildung und Forschung and the European Union Marie-Curie Program (A.S.)

Correspondence should be addressed to Prof. Peter Brown, Sobell Department of Motor Neuroscience and Movement Disorders, Institute of Neurology, Queen Square, London WCIN 3BG, UK. E-mail: p.brown@ion.ac.uk. D0I:10.1523/JNEUROSC1.5295-07.2008

Copyright $\odot 2008$ Society for Neuroscience $\quad 0270-6474 / 08 / 283008-09 \$ 15.00 / 0$ different frequencies. BG activities at frequencies of $20-30 \mathrm{~Hz}$ were coupled with mesial cortical areas such as the supplementary motor area (SMA), but activities at lower frequency were more diffusely coupled with cortex, including lateral cortical motor areas such as the sensorimotor cortex. Moreover, EEG activity was shown to lead BG activity over $20-30 \mathrm{~Hz}$, perhaps through cortico-striatal pathways (Alexander and Crutcher, 1990; Parent and Hazrati, 1995), whereas BG LFP activity could lead EEG activity at lower frequencies. Together, these observations made possible the suggestion that the frequency of synchronization may be exploited as a means of marking and segregating processing in the different functional subloops, over and above any anatomical segregation of processing streams (Fogelson et al., 2006).

However, these coupling studies fail to address two important issues. First, if different functional loops (e.g., those between the BG and lateral and mesial cortical motor areas) are effectively tuned to different, or at least partly different, frequencies, one would predict a differential pattern of modulation of these segregated activities during movement. Second, analyses to date have assumed unidirectional flow of information around BG-cortical loops, whether from or to the cortex. However, more likely is the possibility of bidirectional communication in this looped architecture, as suggested in anesthetized rodents (Sharott et al., 2005). 


\begin{tabular}{|c|c|c|c|c|c|c|c|c|c|c|c|}
\hline \multirow[b]{2}{*}{ Case } & \multirow[b]{2}{*}{ Sex } & \multirow[b]{2}{*}{ Age (years) } & \multirow[b]{2}{*}{$\begin{array}{l}\text { Disease } \\
\text { duration (years) }\end{array}$} & \multirow[b]{2}{*}{ Diagnosis } & \multicolumn{2}{|c|}{$\begin{array}{l}\text { Motor UPDRS, no } \\
\text { DBS (2 d postop) }\end{array}$} & \multicolumn{2}{|c|}{$\begin{array}{l}\text { Motor UPDRS, no MED } \\
\text { (1 year postop) }\end{array}$} & \multirow[b]{2}{*}{ Handedness } & \multicolumn{2}{|c|}{$\begin{array}{l}\text { MED (L-Dopa equivalent } \\
\mathrm{mg} / \mathrm{d} \text { ) }\end{array}$} \\
\hline & & & & & $\begin{array}{l}\text { OFF } \\
\text { MED }\end{array}$ & $\begin{array}{l}\text { ON } \\
\text { MED }\end{array}$ & OFF DBS & ONDBS & & Preop & $\begin{array}{l}6 \text { months } \\
\text { postop }\end{array}$ \\
\hline 1 & $\mathrm{~F}$ & 65 & 15 & Idiopathic PD & 46 & 14 & 46 & 32 & $\mathrm{R}$ & 1400 & 75 \\
\hline 2 & $M$ & 58 & 10 & Idiopathic PD & 29 & 8 & 42 & 12 & $\mathrm{~L}$ & 1550 & 100 \\
\hline 3 & $M$ & 65 & 6 & Idiopathic PD & 20 & 4 & 32 & 12 & $\mathrm{R}$ & 1300 & 800 \\
\hline 4 & $M$ & 51 & 7 & Idiopathic PD & 30 & 4 & 22 & 11 & $\mathrm{R}$ & 1500 & 75 \\
\hline 5 & $\mathrm{~F}$ & 63 & 12 & Idiopathic PD & 34 & 8 & 41 & 26 & $\mathrm{R}$ & 1250 & 1000 \\
\hline 6 & $\mathrm{~F}$ & 55 & 11 & Idiopathic PD & 28 & 5 & 27 & 10 & $\mathrm{R}$ & 800 & 350 \\
\hline 7 & $M$ & 59 & 16 & Idiopathic PD & 20 & 2 & 23 & 8 & $\mathrm{R}$ & 1450 & 250 \\
\hline 8 & $\mathrm{~F}$ & 65 & 8 & Idiopathic PD & 41 & 10 & ND & ND & $\mathrm{R}$ & 600 & 400 \\
\hline 9 & $M$ & 51 & 10 & Idiopathic PD & 27 & 5 & ND & ND & $\mathrm{R}$ & 650 & 200 \\
\hline 10 & M & 59 & 8 & Idiopathic PD & 37 & 8 & ND & ND & $\mathrm{R}$ & 600 & 300 \\
\hline 11 & M & 65 & 8 & Idiopathic PD & $\begin{array}{l}4 \\
1\end{array}$ & 7 & $\begin{array}{l}\text { ND } \\
\text { ND }\end{array}$ & $\begin{array}{l}\text { ND } \\
\text { ND }\end{array}$ & $\mathrm{R}$ & 1900 & 500 \\
\hline $\begin{array}{l}12 \\
\text { Mean } \pm S D\end{array}$ & $M$ & 52 & 25 & PD with Parkin gene mutation & $\begin{array}{l}47 \\
33 \pm 9\end{array}$ & $\begin{array}{r}23 \\
8 \pm 6\end{array}$ & $\begin{array}{l}\text { ND } \\
33 \pm 10\end{array}$ & $\begin{array}{l}\text { ND } \\
16 \pm 9\end{array}$ & $\mathrm{R}$ & $\begin{array}{l}500 \\
1125 \pm 135\end{array}$ & $\begin{array}{l}150 \\
350 \pm 84\end{array}$ \\
\hline
\end{tabular}

All patients underwent bilateral implantation of STN. All had a good levodopa (L-Dopa) response but severe motor complications. F, Female; M, male; postop, postoperative; Preop, preoperative; MED, medication; R, right; L, left; ND, Not determined yet.

Here, we investigate these issues by studying alert PD patients with implanted deep-brain stimulation (DBS) electrodes while they rested or performed a repetitive movement of one upper limb. We then applied the directed transfer function (DTF) to identify bidirectional communication between cortical EEG and BG LFPs (Kaminski and Blinowska, 1991; Cassidy and Brown, 2003). Analyses were focused on how coupling might change during motor processing and how this might be affected by the relative presence or absence of dopaminergic input.

\section{Materials and Methods}

Patients and electrode implantation. All patients gave their informed written consent to participate in this study, which was approved by the ethics committee of the Hospital for Neurology Pierre Wertheimer (Lyon, France), in accordance with The Code of Ethics of the World Medical Association (Declaration of Helsinki, 1967). We studied 12 patients (eight men and four women; mean age, $59 \pm 6$ years; range, 51-65) with PD for 6-25 years (all idiopathic, except one with the Parkin gene mutation). Handedness was assessed using the Edinburgh, Oldfield, Handedness Inventory (Oldfield, 1971), and all patients, except one, were right handed.

Patients underwent bilateral implantation of deep-brain electrodes in the subthalamic nucleus (STN) for the treatment of severe PD (Table 1). All patients had good levodopa responsiveness [mean reduction in the United Parkinson's Disease Rating Scale (UPDRS) motor score by levodopa, 6 months preoperatively: $76 \pm 8 \%$ ] and severe motor complications. The exclusion criteria were as follows: age $\geq 70$ years old, cognitive decline (score $<130$ on the Mattis Dementia Rating Scale), major depression on the Beck Depression Inventory (score $>20$ ), abnormal brain magnetic resonance imaging (MRI), and concomitant severe medical pathologies.

The surgical procedure has been detailed in a previous publication (Thobois et al., 2002). Briefly, STN was located by MRI and ventriculography. Implantation was done under local anesthesia. Microrecordings (Guideline System 3000a; Molecular Devices, Foster City, CA) and macrostimulation were performed to determine the best trajectory among the three tested for each side (Radionics, Burlington, MA). Then, definitive electrodes were implanted bilaterally (model 3389-28; Medtronic, Minneapolis, $\mathrm{MN}$ ). Brain MRI was performed postoperatively to check the final electrode placement and exclude surgical complications. At least one contact was considered to lie in the dorsal STN after postoperative MRI. Six days later, a subcutaneous programmable pulse generator (Itrell II or Kinetra; Medtronic) was implanted under general anesthesia and connected to the implanted electrodes. Seven of the 12 patients have so far reached their 1 year follow-up and were assessed OFF medication/ON and OFF stimulation. In these, UPDRS motor scores exhibited a $54 \pm 16 \%$ decrease from OFF med-
ication/OFF stimulation $(33.3 \pm 10)$ to OFF medication/ON stimulation $(16 \pm 9)$. Across all 12 patients, levodopa equivalent medication fell from $1125 \pm 135 \mathrm{mg}$ (SEM) preoperatively to $350 \pm 84 \mathrm{mg}$ during postoperative follow-up (paired $t$ test: $t_{(11)}=5.10 ; p<0.01$ ). These observations (Table 1) are consistent with adequate targeting.

Recordings and paradigm. Data were collected $5 \mathrm{~d}$ postoperatively in the interval between macroelectrode implantation and subsequent connection to a subcutaneous stimulator. Subjects were comfortably seated in front of a table during a $100 \mathrm{~s}$ rest period and while they made externally paced single taps (alternating extension and flexion at the proximal metocarpophalangeal joint) with the dominant index finger on a custom-made tapping device: taps had to be performed approximately every second after a regular tone given by a metronome. Two sessions of seven series of 20 taps separated by $20 \mathrm{~s}$ rest periods were recorded. Note that the metronome was kept on during the recording performed at rest to avoid any difference in auditory processing between the two conditions. Each recording was performed after overnight withdrawal of antiparkinsonian medication (OFF medication) and $1 \mathrm{~h}$ after administration of the patient's usual morning levodopa equivalent dose plus $25 \%$ (ON medication).

Scalp electroencephalogram was recorded according to the 10:20 international system from an electrode pair over C3 and FC3 in righthanded patients or $\mathrm{C} 4$ and FC4 in the left-handed patient. In addition, we recorded from $\mathrm{Cz}$ and $\mathrm{FCz}$. Note that $\mathrm{C} 3 / 4$ and $\mathrm{Cz}$ approximately overlie the hand area of the sensorimotor cortex, including the primary motor cortex (M1) and the SMA, respectively, although electrodes here will not solely pick-up activities from these areas. EEG electrodes were referenced to linked ears, but a bipolar extraction of $\mathrm{C} 3 / 4-\mathrm{FC} 3 / 4$ and $\mathrm{Cz}-\mathrm{FCz}$ was performed off-line to limit volume conduction between candidate areas. The sparseness of EEG recordings was dictated by surgical dressings.

STN LFPs were recorded from the four contacts of each DBS macroelectrode, referenced to linked ears. Thereafter, we performed an off-line extraction of bipolar signals from adjacent contact pairs 01,12 , and 23, in which contact pair 01 was the deepest. This was also performed to limit volume conduction. Signals were amplified, bandpass filtered between 0.5 and $90 \mathrm{~Hz}$ (6 dB per octave; Biopotential Analyser Diana, St. Petersburg, Russia), and recorded onto a personal computer using custom-written software (A. Pogosyan). Sampling rates were $184 \mathrm{~Hz}$ for five patients, $1000 \mathrm{~Hz}$ for one patient, and $1200 \mathrm{~Hz}$ for six patients. In the latter two instances, data were interpolated off-line to a common sampling frequency of $184 \mathrm{~Hz}$. Overall, the risk of aliasing was very small, given that sampling rates were just above twice or more the steep low-pass filter.

Data analysis. Data were analyzed using Spike2 software (Cambridge Electronic Design, Cambridge, UK) and Matlab (Mathworks, Natick, MA). EEG signals and simultaneously recorded LFP signals were divided into $10 \mathrm{~s}$ segments either at rest or during tapping, when the period 
between 2 and $12 \mathrm{~s}$ after the onset of each tapping series was selected. We analyzed STN LFPs from the contact pair contralateral to the dominant hand that showed the highest $\beta$ band power at rest OFF medication, based on evidence that this activity is generated in dorsolateral "motor" STN (Kühn et al., 2005; Chen et al., 2006; Weinberger et al., 2006; Trottenberg et al., 2007).

Using a multiple autoregressive model (MAR), we calculated signal power at three sites [the lateral sensorimotor cortical area (C3/4-FC3/4, hereafter termed "M1" solely for convenience), the central cortical motor area (Cz-FCz, hereafter termed "SMA" for convenience), and STN] and evaluated the possible DTF based asymmetries of flows between corticalsubcortical sites pairs (M1 to STN, STN to M1, SMA to STN, STN to SMA) for both motor states (rest and during movement) and both dopaminergic states (ON and OFF medication). A detailed description of the methodology and principles of the DTF can be found in the studies by Korzeniewska et al. (2003) and Cassidy and Brown (2003). Briefly, the DTF investigates any possible asymmetry in the flow of coherent activity between sites (Kaminski and Blinowska, 1991). To this end, the MAR model that best described the signals coming from the three sites of interest (M1, SMA, STN) was determined. Unlike power measures, the MAR methodology is essential for calculating the DTF, because the DTF is built directly from the MAR coefficients. Power, however, can also be calculated from the model for comparison with the DTF. Following the procedure detailed by Cassidy and Brown (2003), a Bayesian methodology was applied to estimate the parameters of the autoregressive model. This approach is desirable in that it provides full probabilistic distributions for all of the model parameters. The autoregressive coefficients can be used to construct a bounded, normalized measure (the DTF) that provides information on the effective direction of coupling between each site. In a previous study (Sharott et al., 2005), a Bayesian methodology was also used to calculate the model order. Although model order selection is important per se, it has become clear that within a sensible range it alters the spectra relatively little (Schlögl and Supp, 2006). Given the computational burden of computing the model order for each data epoch, we therefore selected a constant model order of 10 , which is ample for the number of frequency components being investigated (Schlögl and Supp, 2006). Multivariate analysis was performed, which, at any given frequency, consists of directed coherence (dCoh) values giving the coherence in each direction between the three sites. In cases in which multiple channels are analyzed multivariate, compared with bivariate, analysis gives a potentially more accurate result, because all these channels are incorporated in the same MAR model. Indeed, in these cases, bivariate analysis can give confusing results (Kus et al., 2004). For any pair of sites, if the dCoh is significantly greater in one direction, the DTF can be considered "asymmetric" in that direction. Conversely, if the dCoh estimates are not significantly different, the DTF can be considered "symmetric" (Sharott et al., 2005).

Statistics. Power data were logged, and dCoh data were Fisher transformed for subsequent statistical analysis. All statistical analyses were conducted using Statistica version 6 (StatSoft, Tulsa, OK). Log power and transformed dCoh data were averaged across three different frequency bands: sub- $\beta(3-13 \mathrm{~Hz}), \beta(14-35 \mathrm{~Hz})$, and $\gamma(65-90 \mathrm{~Hz})$. These discontinuous frequency bands were selected because several previous studies have shown that spectral peaks and physiologically reactive LFP power in the STN are seen below $35 \mathrm{~Hz}$ and again from $\sim 65-90 \mathrm{~Hz}$ (Brown et al., 2001; Williams et al., 2002; Priori et al., 2004; Alegre et al., 2005; Fogelson et al., 2005; Alonso-Frech et al., 2006; Devos and Defebvre, 2006; Kühn et al., 2006a,b; Androulidakis et al., 2007). The precise frequency range of the $\beta$ band was chosen to match (as far as possible, given differences in sampling rates) the band determined to be focally generated in the STN in recent intraoperative (Kühn et al., 2005; Chen et al., 2006) and postoperative (Kühn et al., 2006a) recordings. This frequency range (14-35 $\mathrm{Hz}$ ) extends a little higher than in previous publications, but the extension of the band is further justified by two studies that include the extent of spectral peaks in individual nuclei. In these, STN LFP spectra in 5 of 16 (Kühn et al., 2004) and 13 of 26 (Doyle et al., 2005) nuclei had peaks involving or extending into 31-35 Hz. Similarly, although previous publications defined the $\gamma$ band as extending to $80 \mathrm{~Hz}$ (Alonso-Frech et al., 2006) or $85 \mathrm{~Hz}$ (Williams et al., 2002; Silberstein et al., 2003), a more recent report demonstrated that spectral peaks in the LFP of PD patients treated with levodopa in the range of $62.3-93 \mathrm{~Hz}$ (Trottenberg et al., 2006). The definition of a broad "sub- $\beta$ band" ( $3-13 \mathrm{~Hz})$ that includes $\theta$ and $\alpha$ bands was motivated by the need to preserve statistical power.

For each brain site, we separately analyzed log power data using a $3 \times$ $2 \times 2$ ANOVA with repeated measures on all factors: frequency band (sub- $\beta, \beta, \gamma) \times$ dopaminergic state $(\mathrm{ON}$ vs $\mathrm{OFF}) \times$ motor state (rest vs movement).

With respect to the DTF, we first checked whether experimental dCoh in each direction was significantly different to that derived from shuffled data. These control data sets (in which no significant flow is expected between all three brain regions) resulted from the randomization of each series of STN and EEG values recorded for each patient, in both dopaminergic states at rest and during movement. dCoh data were first examined with six $2 \times 2 \times 2 \times 2$ ANOVAS with repeated measures on all factors: dopaminergic state $(\mathrm{ON}$ vs $\mathrm{OFF}) \times$ motor state (rest vs movement) $\times$ flow direction (cortex:STN vs STN:cortex) $\times$ shuffled state (experimental or unshuffled data vs control or shuffled data). All ANOVAs were separately applied for each of the three frequency bands (sub- $\beta$, $\beta, \gamma)$ and cortico-subcortical pairs of sites (M1/STN, SMA/STN). These ANOVAs were principally performed to establish whether experimental dCoh exceeded control (shuffled) dCoh but also demonstrated whether there were differences between conditions.

Second, we performed two $3 \times 2 \times 2$ ANOVAs with repeated measures on all factors: frequency band (sub- $\beta$ vs $\beta$ vs $\gamma$ ) $\times$ flow direction (cortex:STN vs STN:cortex) $\times$ pair of brain sites (M1/STN, SMA/STN) at rest for each dopaminergic state (ON and OFF). These ANOVAs allowed a comparison across bands and pairs of sites. Note that we did not explore dCoh between mesial and lateral electroencephalography from the multivariate analysis, because this was likely to be biased by volume conduction effects and so as not to weaken statistical analyses through the inclusion of too many factors.

Data entered in ANOVAs were nonspherical (as determined by Mauchly's tests), so we applied Greenhouse-Geisser corrections to the resulting $p$ values. We used Fisher least significant difference post hoc tests where appropriate. Corrected $p$ values $\leq 0.05$ were considered significant, although those near 0.05 should be interpreted with caution.

\section{Results \\ Power}

Power at each site was affected by frequency (M1, $p<0.01$; SMA, $p<0.01$; STN, $p<0.01$ ), whereby power in the $\gamma$ band was less than that in the sub- $\beta$ and $\beta$ bands ( $p o s t$ hoc test, $p<0.05$ ) (Fig. 1 , Table 2). Surprisingly, there were no effects of dopaminergic state, but in the cases of SMA and STN, there were interactions between frequency band and motor state (SMA, $p=0.03$; STN, $p=0.03$ ). These were attributable to higher power in the $\gamma$ band during movement than at rest $(p<0.05)$.

\section{dCoh}

Significant features in the pattern of dCoh (Fig. 2; Tables 3, 4) are summarized in the schema illustrated in Figure 3, which shows that all cortico-subcortical flows were greater than shuffled data, whatever the direction, dCoh could be greater at rest than during movement in the $\beta$ band, and dCoh between SMA and STN and from M1 to STN was higher ON than OFF drugs in the $\gamma$ band. All ANOVA results are summarized in Tables 3 and 4 , and those that were significant are detailed below together with relevant post hoc tests.

\section{Differences in dCoh from control data and \\ between conditions}

The dCoh between cortex and STN in the sub- $\beta$ band depended on whether data were shuffled or not, with experimental dCoh higher than shuffled dCoh, whatever the cortico-subcortical pair considered (M1/STN, $p=0.03$; SMA/STN, $p<0.01$ ) (Table 3).

In the $\beta$ band (Fig. $2 A$ ), the dCoh between M1 and STN was 


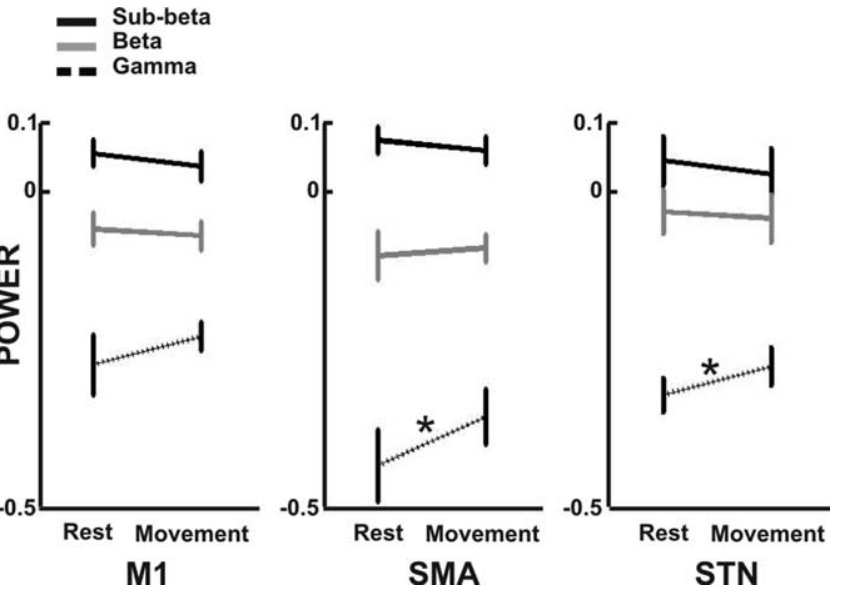

Figure 1. Group power means modulated by motor state and frequency band. Averages across all patients ( $n=12$ ) of Log power measured at the three brain sites considered (M1, SMA, and STN) at rest and during movement in the three frequency bands (continuous black line, sub- $\beta$; gray line, $\beta$; dashed black line, $\gamma$ ) are shown. The three frequency band $X$ dopaminergic state $\times$ motor state ANOVAs performed for each brain site (M1, SMA, STN) showed that although there was only a difference of power attributable to the frequency band at M1 $(p<0.01)$, with power in the $\gamma$ band lower than those in the lower-frequency bands, the motor state interacted with this factor at SMA and STN $(p<0.05)$ : in the two latter cases, power was increased from rest to movement $(p<0.05)$ in the $\gamma$ band only. There was also a rise of power as frequency dropped ( $p<0.05$ ). The asterisk denotes a significant effect of motor state on power. Unexpectedly, power was not affected by dopaminergic state. Note that here and in ensuing figures, M1 and SMA are used to denote lateral and mesial cortical motor sites as detected with electroencephalography from $\mathrm{C} 3 / 4-\mathrm{FC} 3 / 4$ and $\mathrm{Cz}-\mathrm{FC}$, respectively.

affected by shuffling ( $p=0.01$ ), experimental dCoh was higher than shuffled dCoh, and there was a trend for an effect of motor state $(p=0.07)$. The dCoh between SMA and STN was affected by motor state $(p=0.04)$, flow direction $(p<0.01)$, and shuffling $(p<0.01)$ with interactions between motor state and shuffling $(p=0.04)$ and between flow direction and shuffling $(p=$ $0.01)$. The dCoh was higher at rest than during movement and in the experimental data compared with the shuffled data. Post hoc results revealed that experimental dCoh was higher from SMA to STN than in the opposite direction $(p<0.05)$.

In the $\gamma$ band (Fig. $2 B$ ), the dCoh between M1 and STN was affected by shuffling ( $p<0.01$ ), with dCoh higher in the experimental condition compared with the shuffled one. There was also an interaction between dopaminergic state, flow direction, and shuffling ( $p=0.05$ ) which was attributable to the fact that, when experimental, the dCoh from M1 to STN was dominant in the ON state $(p<0.05)$. The dCoh between the SMA and STN was affected by dopaminergic state $(p=0.03)$ and shuffling $(p<$ $0.01)$, and there was an interaction between these two factors $(p=0.02)$. Overall, post hoc tests demonstrated that dCoh between SMA and STN was higher, when experimental, in the ON state compared with the OFF state, whatever the flow direction and the motor state $(p<0.05)$.

\section{Differences in dCoh between bands and pairs of brain sites}

The dCoh data in the OFF state at REST revealed a trend for an effect of frequency band ( $p=0.06)$ and interactions between flow direction and pair of sites $(p=0.01)$ and between frequency band, flow direction, and pair of brain sites $(p=0.04)$ (Fig. $2 C$, Table 4). Post hoc analyses revealed that in the sub- $\beta$ band, dCoh was higher from cortex to STN than in the opposite direction $(p<0.05)$. However, there was no difference in $\mathrm{dCoh}$ between each pair of sites. In the $\beta$ band, flows from cortex were greater than flows from STN and, although there was no difference in dCoh for flow going from STN either to M1 or SMA, flows going from SMA to STN dominated flows going from M1 to STN ( $p<$ $0.05)$. There was no such difference in the $\gamma$ band. Also, flows going from cortex to STN were more pronounced in the sub- $\beta$ and $\beta$ bands than in the $\gamma$ band $(p<0.05)$, and when originating in SMA, they were higher in the $\beta$ band than in the sub- $\beta$ band $(p<0.05)$. In contrast, there was no difference in dCoh attributable to frequency band in flows going from STN to cortex.

In the ON state at rest (Fig. $2 C$ ), dCoh was affected by frequency band $(p=0.01)$. In addition, there were interactions between frequency band and flow direction $(p=0.04)$, between flow direction and site pair $(p=0.04)$, as well as between frequency band, flow direction, and site pair $(p=0.01)$. Overall, post hoc tests $(p<0.05)$ showed that in the sub- $\beta$ band, dCoh was dominant when flow was coming from SMA rather than in the opposite direction, but no such difference was detected for the M1/STN pair. In the $\beta$ band, dCoh was superior for flow coming from rather than going to cortex, and as in the OFF state, $\mathrm{dCoh}$ was greater for flows coming from SMA than those coming from M1. Again, there was no difference in the $\gamma$ band. Finally, $\mathrm{dCoh}$ from cortex to STN in the $\beta$ band was higher than in the $\gamma$ band, whatever the pair considered, whereas dCoh in the sub- $\beta$ band was lower than in the $\beta$ band and higher than in the $\gamma$ band for the SMA/STN pair only. No difference attributable to frequency band was found for flows coming from STN.

Finally, $\mathrm{dCOH}$ from cortex to STN obtained in the $\beta$ band was higher than the one found in the $\gamma$ band, whatever the pair considered, whereas dCoh in the sub- $\beta$ band was between those in $\beta$ and $\gamma$ bands for the SMA/STN pair only. No difference attributable to frequency band was found for flows coming from STN.

\section{Discussion}

The BG are anatomically situated at the center of loops between the cortex and the thalamus. Despite this, the interplay between cortex and BG, which is likely to underlie both the physiological and pathological roles of these networks, is often ignored in electrophysiological studies. The data presented here provide the first investigation into directional interactions between the human cortex and STN under differing levels of dopamine and dynamic movement. The results suggest several organizational principles in STN-cortical circuits.

First, experimental dCoh exceeded control shuffled data, whatever the frequency band and the pair of brain sites considered. This suggests a significant connectivity with bidirectional, rather than unidirectional, flows of information across a very broad range of frequencies within STN subloops involving lateral and mesial cortical motor areas. Second, these subloops are not identical in their precise pattern of information flow. In the $\beta$ band, more information flowed from SMA to STN than from M1 to STN, regardless of drug state. Third, there is some frequencydependent modulation of information flow according to dopaminergic state and the presence of voluntary movement. Information flows between SMA and STN were greater at rest than during movement in the $\beta$ band. In addition, information flows between SMA and STN and from M1 to STN were higher ON than OFF drugs in the $\gamma$ band. These effects could not be explained by changes in EEG or LFP power.

The generic limitations of this kind of study have been discussed previously, including the presumptive localization of the analyzed contacts in the STN and the far from perfect localization of cortical generators (Williams et al., 2002; Fogelson et al., 2006). A surprising finding contradicting previous reports was the fail- 
Table 2. Statistical results of power: dopaminergic state "motor state" frequency-band ANOVAs

\begin{tabular}{|c|c|c|c|c|c|c|c|c|c|}
\hline \multirow[b]{2}{*}{ Effect } & \multicolumn{3}{|l|}{ M1 } & \multicolumn{3}{|c|}{ SMA } & \multicolumn{3}{|c|}{ STN } \\
\hline & df & $F$ & Corrected $p$ & df & $F$ & Corrected $p$ & $d f$ & $F$ & Corrected $p$ \\
\hline Dopaminergic state (F1) & 1 & 1.77 & 0.21 & 1 & 1.52 & 0.24 & 1 & 0.02 & 0.89 \\
\hline Error & 11 & & & 11 & & & 11 & & \\
\hline Motor state (F2) & 1 & 0.10 & 0.76 & 1 & 1.15 & 0.31 & 1 & 0.53 & 0.48 \\
\hline Error & 11 & & & 11 & & & 11 & & \\
\hline Frequency band (F3) & 2 & 39.86 & $\underline{0.00}$ & 2 & 46.63 & $\underline{0.00}$ & 2 & 69.02 & $\underline{0.00}$ \\
\hline Error & 22 & & & 22 & & & 22 & & \\
\hline $\mathrm{F} 1{ }^{*} \mathrm{~F} 2$ & 1 & 0.51 & 0.49 & 1 & 0.08 & 0.78 & 1 & 0.16 & 0.70 \\
\hline Error & 11 & & & 11 & & & 11 & & \\
\hline $\mathrm{F} 1 * \mathrm{~F} 3$ & 2 & 1.02 & 0.35 & 2 & 0.77 & 0.42 & 2 & 1.54 & 0.24 \\
\hline Error & 22 & & & 22 & & & 22 & & \\
\hline $\mathrm{F} 2 * \mathrm{~F} 3$ & 2 & 2.24 & 0.16 & 2 & 4.90 & $\underline{0.03}$ & 2 & 5.89 & $\underline{0.02}$ \\
\hline Error & 22 & & & 22 & & & 22 & & \\
\hline $\mathrm{F} 1{ }^{*} \mathrm{~F} 2{ }^{*} \mathrm{~F} 3$ & 2 & 0.59 & 0.52 & 2 & 0.36 & 0.58 & 2 & 1.03 & 0.35 \\
\hline Error & 22 & & & 22 & & & 22 & & \\
\hline
\end{tabular}

Original degrees of freedom (df), Fvalues, and Greenhouse-Geisser corrected $p$ values are given for each ANOVA performed on logged power or dCoh data. Significant results with probability strictly below 0.05 are presented underlined. For clarity, results are presented with original degrees of freedom and $p$ values rounded to two decimal places.

ure to demonstrate a change in power in the STN LFP because of drug state. This suggests that the effects of levodopa challenges might have been suboptimal compared with previous studies in which a suppression of $\beta$ band LFP power (Priori et al., 2004; Alonso-Frech et al., 2006; Kühn et al., 2006b) and an increase in $\gamma$ band LFP power (Williams et al., 2002; Alegre et al., 2005; Fogelson et al., 2005; Alonso-Frech et al., 2006; Devos and Defebvre, 2006) have been reported. LFP recordings were consistently performed on the fifth day postoperatively, and it is possible that levodopa effects were attenuated by microlesional effects, especially postoperative edema, at this time. Insensitivity of our MAR model to power changes seems an unlikely explanation for the failure to demonstrate a change in power in the STN LFP because of drug state, because this negative result was corroborated by Fourier analysis of our data set (results not shown). Regardless, the absence of an effect of medication on LFP power suggests that this study might underestimate changes in information flow between cortex and STN attributable to dopaminergic input.

The inferences possible from dCoh also deserve comment. dCoh implies that covariance occurs in phase and amplitude between two signals and that such coupled activity is "driven" from one of the signals to the other. In so far as coherence may be viewed in terms of functional connectivity (Thatcher et al., 1986; Rappelsberger and Petsche, 1988; Shen et al., 1999; Buzsáki and Draguhn, 2004; Magill et al., 2006), the dCoh measure can be thought of as describing possible paths of information flow underpinning this connectivity. However, whether these paths are plausible depends on anatomical connectivity and adequate sampling of sites involved in any given interaction. Certainly, there are direct and indirect cortical inputs to STN and indirect projections from
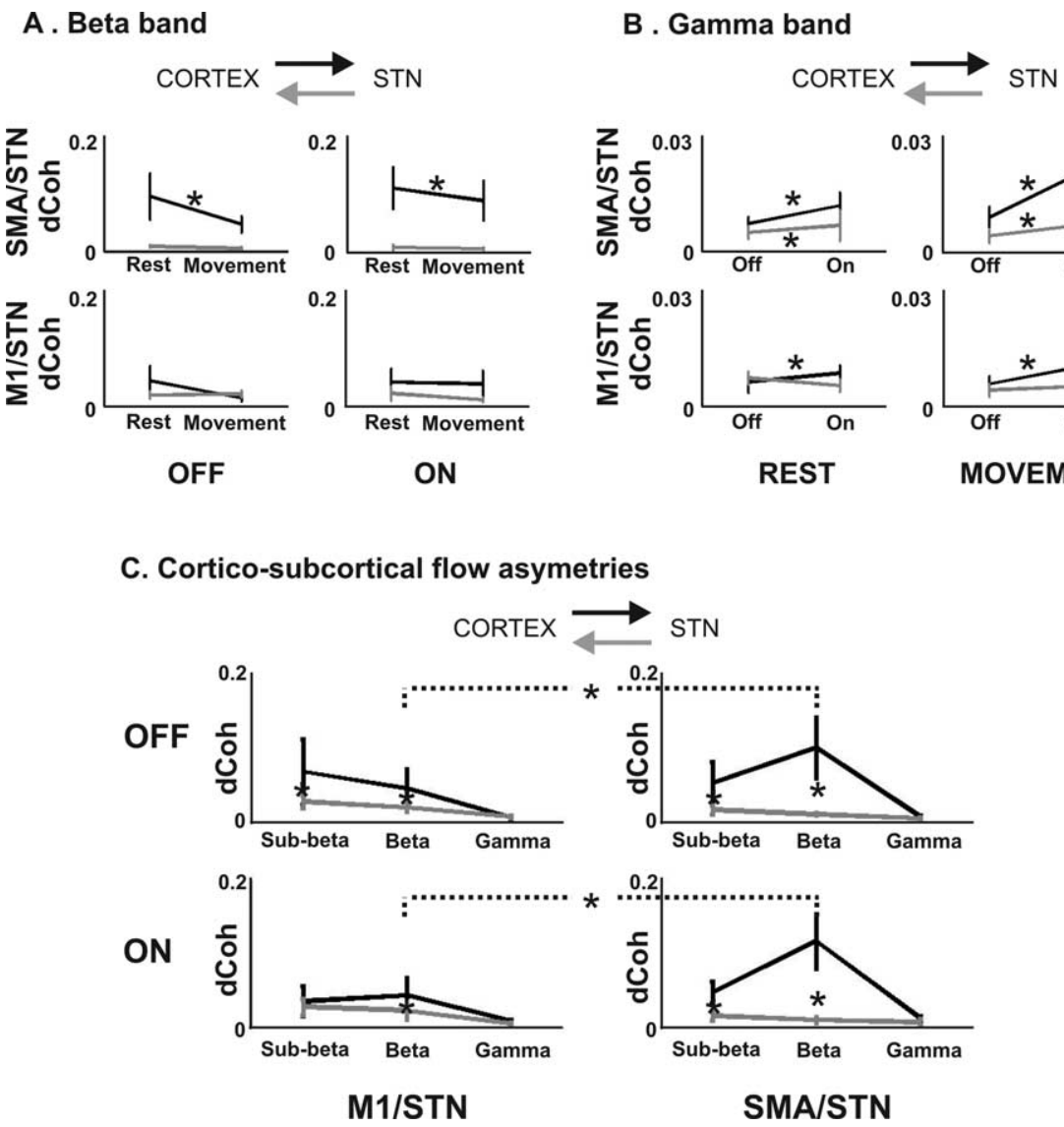

Figure 2. dCoh asymmetries modulated by motor state and dopaminergic state. Averages across all patients $(n=12)$ of $d C o h$ between SMA and STN or between M1 and STN according to the flow direction (black lines, cortex to STN; gray lines, STN to cortex), motor state (at rest or during movement), and dopaminergic state (OFF or $0 N$ ) are shown. $A$, In the $\beta$ band, there is a drop in flows going from SMA to STN with movement (ANOVAs with repeated measures, ${ }^{*} p<0.05$ ). $\boldsymbol{B}$, Flows in the $\gamma$ band increase between SMA and STN and from M1 to STN when on dopaminergic treatment (ANOVAs with repeated measures, ${ }^{*} p<0.05$ ). $C$, Flow asymmetries at rest in the OFF and ON states according to the frequency band and the pair of brain sites considered. There are greater flows from cortex to STN (black line) than in the opposite direction (gray line), particularly for flow coming from SMA. ANOVAs with repeated measures, ${ }^{*} p<0.05$ for comparisons between frequency bands and pairs of brain sites.

STN to cortex that might underlie the coupling described here (Alexander and Crutcher, 1990; Parent and Hazrati, 1995; Maurice et al., 1998; MacKinnon et al., 2005), but sampling of structures was inevitably restricted, given our recordings in patients. Nevertheless, because of this, we cannot exclude that dCoh might have arisen as a 
Table 3. Statistical results of dCoh: dopaminergic state "motor state" direction flow shuffled state ANOVAs

\begin{tabular}{|c|c|c|c|c|c|c|c|c|c|c|c|c|c|c|c|c|c|c|}
\hline \multirow[b]{3}{*}{ Effect } & \multicolumn{6}{|c|}{ Sub- $\beta$} & \multicolumn{6}{|l|}{$\beta$} & \multicolumn{6}{|l|}{$\gamma$} \\
\hline & \multicolumn{3}{|c|}{ M1/STN } & \multicolumn{3}{|c|}{ SMA/STN } & \multicolumn{3}{|c|}{ M1/STN } & \multicolumn{3}{|c|}{ SMA/STN } & \multicolumn{3}{|c|}{ M1/STN } & \multicolumn{3}{|c|}{ SMA/STN } \\
\hline & $d f$ & $F$ & Corrected $p$ & df & $F$ & Corrected $p$ & df & $F$ & Corrected $p$ & $d f$ & $F$ & Corrected $p$ & $d f$ & $F$ & Corrected $p$ & df & $F$ & Corrected $p$ \\
\hline Dopaminergic state (F1) & 1 & 0.12 & 0.73 & 1 & 0.77 & 0.40 & 1 & 0.21 & 0.66 & 1 & 0.71 & 0.42 & 1 & 2.81 & 0.12 & 1 & 6.19 & $\underline{0.03}$ \\
\hline Error & 11 & & & 11 & & & 11 & & & 11 & & & 11 & & & 11 & & \\
\hline Motor state (F2) & 1 & 0.60 & 0.45 & 1 & 0.01 & 0.92 & 1 & 4.09 & 0.07 & 1 & 5.46 & $\underline{0.04}$ & 1 & 0.30 & 0.59 & 1 & 1.11 & 0.31 \\
\hline Error & 11 & & & 11 & & & 11 & & & 11 & & & 11 & & & 11 & & \\
\hline Direction flow (F3) & 1 & 0.77 & 0.40 & 1 & 2.91 & 0.12 & 1 & 0.91 & 0.36 & 1 & 10.46 & 0.01 & 1 & 0.77 & 0.40 & 1 & 2.30 & 0.16 \\
\hline Error & 11 & & & 11 & & & 11 & & & 11 & & & 11 & & & 11 & & \\
\hline Shuffled state (F4) & 1 & 6.39 & $\underline{0.03}$ & 1 & 14.35 & $\underline{0.00}$ & 1 & 8.89 & $\underline{0.01}$ & 1 & 16.97 & $\underline{0.00}$ & 1 & 43.00 & $\underline{0.00}$ & 1 & 32.59 & $\underline{0.00}$ \\
\hline Error & 11 & & & 11 & & & 11 & & & 11 & & & 11 & & & 11 & & \\
\hline $\mathrm{F} 1{ }^{*} \mathrm{~F} 2$ & 1 & 1.05 & 0.33 & 1 & 1.18 & 0.30 & 1 & 0.23 & 0.64 & 1 & 0.48 & 0.50 & 1 & 0.95 & 0.35 & 1 & 2.07 & 0.18 \\
\hline Error & 11 & & & 11 & & & 11 & & & 11 & & & 11 & & & 11 & & \\
\hline $\mathrm{F} 1{ }^{*} \mathrm{~F} 3$ & 1 & 0.06 & 0.81 & 1 & 0.77 & 0.40 & 1 & 0.52 & 0.49 & 1 & 0.64 & 0.44 & 1 & 4.56 & 0.06 & 1 & 2.03 & 0.18 \\
\hline Error & 11 & & & 11 & & & 11 & & & 11 & & & 11 & & & 11 & & \\
\hline $\mathrm{F} 2{ }^{*} \mathrm{~F} 3$ & 1 & 0.34 & 0.57 & 1 & 0.05 & 0.82 & 1 & 1.24 & 0.29 & 1 & 3.65 & 0.08 & 1 & 0.56 & 0.47 & 1 & 2.90 & 0.12 \\
\hline Error & 11 & & & 11 & & & 11 & & & 11 & & & 11 & & & 11 & & \\
\hline $\mathrm{F} 1{ }^{*} \mathrm{~F} 4$ & 1 & 0.12 & 0.73 & 1 & 0.73 & 0.41 & 1 & 0.19 & 0.67 & 1 & 0.70 & 0.42 & 1 & 3.29 & 0.10 & 1 & 7.93 & $\underline{0.02}$ \\
\hline Error & 11 & & & 11 & & & 11 & & & 11 & & & 11 & & & 11 & & \\
\hline $\mathrm{F} 2{ }^{*} \mathrm{~F} 4$ & 1 & 0.60 & 0.45 & 1 & 0.01 & 0.92 & 1 & 3.82 & 0.08 & 1 & 5.43 & $\underline{0.04}$ & 1 & 0.09 & 0.77 & 1 & 1.31 & 0.28 \\
\hline Error & 11 & & & 11 & & & 11 & & & 11 & & & 11 & & & 11 & & \\
\hline $\mathrm{F}^{*} \mathrm{~F} 4$ & 1 & 0.76 & 0.40 & 1 & 2.84 & 0.12 & 1 & 0.91 & 0.36 & 1 & 10.44 & $\underline{0.01}$ & 1 & 0.75 & 0.41 & 1 & 1.99 & 0.19 \\
\hline Error & 11 & & & 11 & & & 11 & & & 11 & & & 11 & & & 11 & & \\
\hline $\mathrm{F} 1{ }^{*} \mathrm{~F} 2{ }^{*} \mathrm{~F} 3$ & 1 & 1.63 & 0.23 & 1 & 1.04 & 0.33 & 1 & 1.57 & 0.24 & 1 & 0.36 & 0.56 & 1 & 0.03 & 0.87 & 1 & 1.83 & 0.20 \\
\hline Error & 11 & & & 11 & & & 11 & & & 11 & & & 11 & & & 11 & & \\
\hline $\mathrm{F} 1{ }^{*} \mathrm{~F} 2{ }^{*} \mathrm{~F} 4$ & 1 & 1.04 & 0.33 & 1 & 1.19 & 0.30 & 1 & 0.25 & 0.63 & 1 & 0.48 & 0.50 & 1 & 1.00 & 0.34 & 1 & 2.01 & 0.18 \\
\hline Error & 11 & & & 11 & & & 11 & & & 11 & & & 11 & & & 11 & & \\
\hline $\mathrm{F} 1{ }^{*} \mathrm{~F} 3{ }^{*} \mathrm{~F} 4$ & 1 & 0.07 & 0.80 & 1 & 0.76 & 0.40 & 1 & 0.56 & 0.47 & 1 & 0.65 & 0.44 & 1 & 5.02 & $\underline{0.05}$ & 1 & 2.41 & 0.15 \\
\hline Error & 11 & & & 11 & & & 11 & & & 11 & & & 11 & & & 11 & & \\
\hline $\mathrm{F} 2{ }^{*} \mathrm{~F} 3{ }^{*} \mathrm{~F} 4$ & 1 & 0.31 & 0.59 & 1 & 0.07 & 0.80 & 1 & 1.23 & 0.29 & 1 & 3.71 & 0.08 & 1 & 0.68 & 0.43 & 1 & 3.57 & 0.09 \\
\hline Error & 11 & & & 11 & & & 11 & & & 11 & & & 11 & & & 11 & & \\
\hline $\mathrm{F} 1{ }^{*} \mathrm{~F} 2{ }^{*} \mathrm{~F} 3{ }^{*} \mathrm{~F} 4$ & 1 & 1.71 & 0.22 & 1 & 1.05 & 0.33 & 1 & 1.48 & 0.25 & 1 & 0.35 & 0.56 & 1 & 0.01 & 0.93 & 1 & 1.47 & 0.25 \\
\hline Error & 11 & & & 11 & & & 11 & & & 11 & & & 11 & & & 11 & & \\
\hline
\end{tabular}

Original degrees of freedom (df), Fvalues, and Greenhouse-Geisser corrected $p$ values are given for each ANOVA performed on logged power or dCoh data. Significant results with probability strictly below 0.05 are presented underlined. For clarity, results are presented with original degrees of freedom and $p$ values rounded to two decimal places.

Table 4. Statistical results of $d$ Coh: frequency band $\times$ direction flow $\times$ pair of sites ANOVAs

\begin{tabular}{|c|c|c|c|c|c|c|}
\hline \multirow[b]{2}{*}{ Dopaminergic state } & \multicolumn{3}{|c|}{ OFF } & \multicolumn{3}{|l|}{ ON } \\
\hline & df & $F$ & Corrected $p$ & $\mathrm{df}$ & $F$ & Corrected $p$ \\
\hline Frequency band (F1) & 2 & 3.98 & 0.06 & 2 & 8.37 & $\underline{0.01}$ \\
\hline Error & 22 & & & 22 & & \\
\hline Direction flow (F2) & 1 & 1.92 & 0.19 & 1 & 3.41 & 0.09 \\
\hline Error & 11 & & & 11 & & \\
\hline Pair of sites (F3) & 1 & 0.58 & 0.46 & 1 & 1.66 & 0.22 \\
\hline Error & 11 & & & 11 & & \\
\hline $\mathrm{F} 1 * \mathrm{~F} 2$ & 2 & 2.33 & 0.15 & 2 & 4.88 & $\underline{0.04}$ \\
\hline Error & 22 & & & 22 & & \\
\hline $\mathrm{F} 1 * \mathrm{~F} 3$ & 2 & 4.07 & 0.05 & 2 & 2.64 & 0.11 \\
\hline Error & 22 & & & 22 & & \\
\hline $\mathrm{F} 2 * \mathrm{~F} 3$ & 1 & 8.74 & $\underline{0.01}$ & 1 & 5.15 & $\underline{0.04}$ \\
\hline Error & 11 & & & 11 & & \\
\hline $\mathrm{F} 1 * \mathrm{~F} 2 * \mathrm{~F} 3$ & 2 & 4.72 & 0.04 & 2 & 6.00 & $\underline{0.01}$ \\
\hline Error & 22 & & & 22 & & \\
\hline
\end{tabular}

Original degrees of freedom $(\mathrm{df}), F$ values, and Greenhouse-Geisser corrected $p$ values are given for each ANOVA performed on logged power or dCoh data. Significant results with probability strictly below 0.05 are presented underlined. For clarity, results are presented with original degrees of freedom and $p$ values rounded to two decimal places.

result of common input to cortex and STN from a third unsampled structure, without any causal influence between the two sampled sites (Sharott et al., 2005).

Given the above limitations, it is important to consider the present results in the context of related findings. To date, most depth recordings in patients with $\mathrm{PD}$ have examined the power of oscilla- tory activity in the STN LFP. This represents synchronization across local populations of neurons rather than synchronization across structures (Hammond et al., 2007). In this study, we did not observe a suppression of synchronization in the $\beta$ band with movement or dopaminergic treatment, as mentioned above, nor an increase in power in the $\gamma$ band with levodopa. However, we clearly observed a rise in $\gamma$ power with movement, as reported previously (Cassidy et al., 2002; Alonso-Frech et al., 2006; Devos and Defebvre, 2006; Androulidakis et al., 2007).

In the case of subcortico-cortical coupling, our findings related to the DTF between cortex and STN are broadly consistent with those of previous studies that have investigated coherence and phase relationships between STN and cortex in patients with PD at rest. Both data sets indicate a greater coupling in the $\beta$ band between STN and mesial rather than lateral motor cortical areas (Fogelson et al., 2006), an increase of coupling in the $\gamma$ band after levodopa (Williams et al., 2002), and a driving of STN activity in the $\beta$ band by cortex (Brown et al., 2001; Marsden et al., 2001; Williams et al., 2002; Fogelson et al., 2006). However, unlike our DTF results, phase estimates have tended to show STN leading cortex in the $\gamma$ band (Williams et al., 2002) and mixed relationships in the sub- $\beta$ band (Williams et al., 2002; Fogelson et al., 2006). In this regard, it is important to note that phase estimates may be ambiguous in systems with bidirectional coupling, such as BG-cortical loops (Cassidy and Brown, 2003). In contrast, the one study investigating dCoh between STN and cortex, albeit in the anesthe- 


\section{OFF}

\section{Sub-beta}
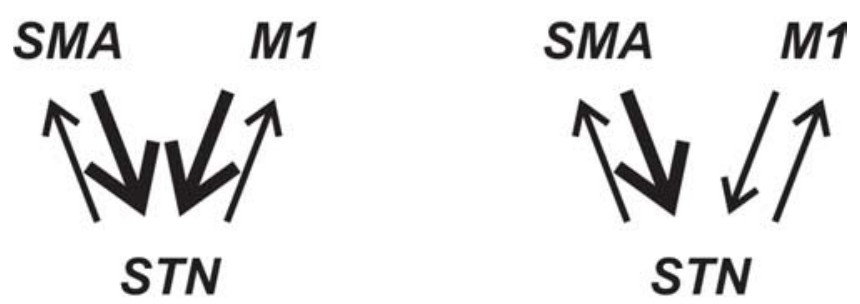

Beta
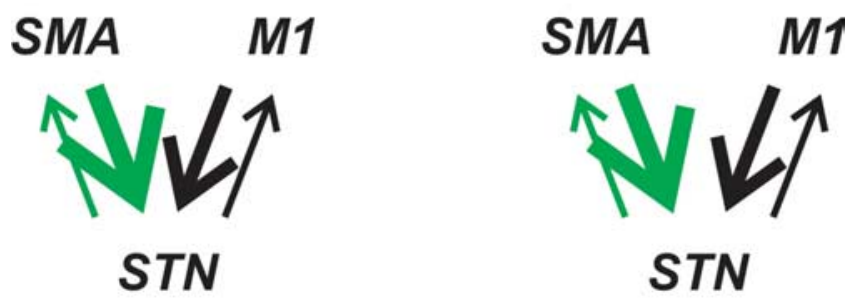

\section{Gamma}
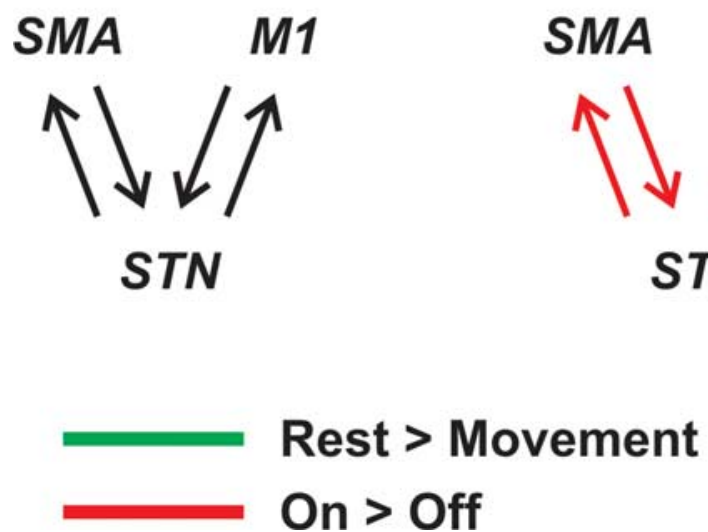

Figure 3. Schematic summary of differences in dCohs. Basic results are drawn from ANOVAs of (frequency band) $\times$ (direction flow) $\times$ (pair of brain sites) performed separately for $0 \mathrm{~N}$ and OFF drugs at rest. Significant asymmetries are shown by the relative thickness of lines. Additional ANOVAs of (drug state) $\times$ (motor state) $\times$ (direction flow) $\times$ (shuffled state) were performed separately for each frequency band and pair of sites. These demonstrated that all arrowed flows were greater than shuffled data, that $\mathrm{dC}$ Ch between SMA and STN were greater at rest than during movement in the $\beta$ band ( $14-35 \mathrm{~Hz}$; with a nonsignificant trend for a similar effect of motor state between M1 and STN), and that dCoh between SMA and STN and that from M1 to STN were higher ON than OFF drugs in the $\gamma$ band $(65-90 \mathrm{~Hz})$.

tized, healthy rodent, demonstrated a net cortical driving of STN activity at frequencies below $60 \mathrm{~Hz}$, rather similar to the present findings (Sharott et al., 2005).

Finally, our findings are in agreement with the only two studies that have commented on changes in coherence between STN LFP and mesial cortical electroencephalography during phasic movement (Cassidy et al., 2002; Kühn et al., 2006a). These studies reported that coherence in the $\beta$ band was attenuated compared with rest, in line with the drop of flows between SMA and STN in the present work. Interestingly, one of these studies also found $\beta$ band coherence to be suppressed during imagination of movement, suggesting that this suppression is related to processing common to movement and motor imagination and not attributable to peripheral feedback (Kühn et al., 2006a).

We found relatively strong bidirectional dCoh between STN and EEG. There is no way of presently establishing whether this coupling is physiological or related to the pathophysiology of PD. However, the results of nonhuman studies and of pharmacological studies in patients suggests that the coherence between the STN and EEG in the sub- $\beta$ and $\beta$ band represented, at the very least, a pathological exaggeration of physiological activity (for review, see Brown and Williams, 2005; Hammond et al., 2007). The picture is less clear for the dCoh in the $\gamma$ band. This was increased rather than suppressed by dopaminergic treatment, and elsewhere, this oscillatory activity has been shown to be lateralized in keeping with similar spectral activity in the nonparkinsonian subject (Androulidakis et al., 2007).

It has been argued previously that synchronization within the BG-cortical loop in the $\beta$ band is essentially antikinetic, favoring postural control over processing related to movement, whereas that in the $\gamma$ band is promoted by dopamine and prokinetic, facilitating movement related processing (Brown, 2003; Gilbertson et al., 2005). Although this schema is likely to be a gross simplification, which in particular assumes homology across activities of different frequencies within a given broad band, it does capture the general changes in information flow observed in the present study. Interestingly, these changes in synchronization across structures occurred in the absence of significant changes in power in the present data set. During movement, there is a suppression of bidirectional coupling between cortical areas and STN in the $\beta$ band. Because there was no significant change in power in the $\beta$ band, the reduction in coupling likely represents a genuine shrinkage in the number of neurons coupled across the two levels (Fig. 4). Conversely, there was an increase in flows between mesial cortical areas and STN and from M1 to STN in the $\gamma$ band after treatment with levodopa, although power in this band did not change with treatment. Accordingly, this implies an increase in the number of neurons coupled across the two levels. In practice, this increase may have been greater during movement than at rest at the subthalamic level, because $\gamma$ STN LFP power was increased during movement (Fig. 4). In summary, there may be reciprocal changes in the number of neuronal elements coupled in the $\beta$ and $\gamma$ frequency bands during movement, paralleling the reciprocity in changes in synchronization evident at the local level (Fogelson et al., 2006) and consistent with the general hypothesis that synchronization in the $\beta$ and $\gamma$ bands within and across structures in the BG-cortical loop impedes and favors processing related to movement, respectively.

The current study is important in demonstrating bidirectional information flow between the cortex and BG in patients with PD, which varies across frequency bands according to dopaminergic and motor state. The findings enrich the model of multiple functional loops between cerebral cortex and STN tuned to different frequencies (Fogelson et al., 2006) and highlight the dynamic nature of functional connectivity through the establishment and deestablishment of extended assemblies of synchronized neurons. 


\section{Beta}

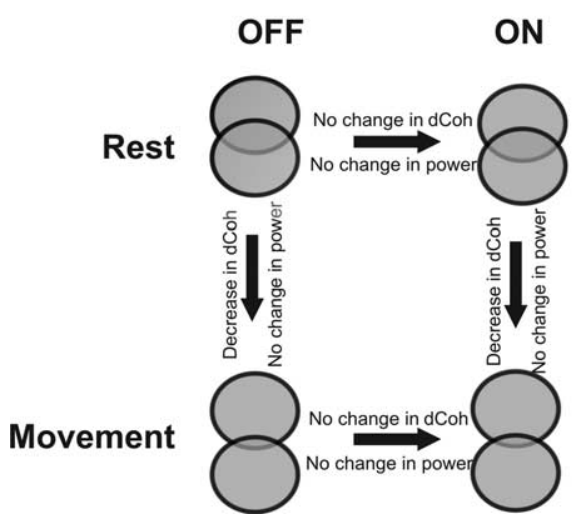

Gamma

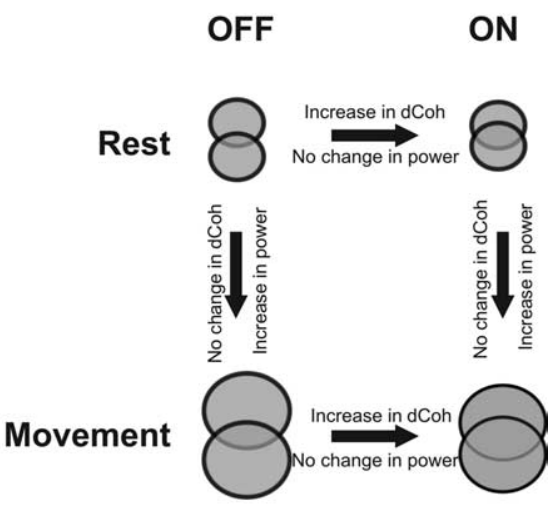

Figure 4. Schematic summary of changes in size of coupled neuronal populations in $\beta$ and $\gamma$ bands. Circles represent mesial motor cortex and STN. The area of circles reflects the number of neuronal elements synchronized and oscillating within a given frequency band (indexed by power), whereas surface area of overlap reflects the number of neuronal elements with coupled activity between mesial motor cortex and STN. The population of neuronal elements coupled in the $\beta$ band $(14-35 \mathrm{~Hz})$ falls during movement, regardless of drug state. The population of neuronal elements coupled in the $\gamma$ band $(65-90 \mathrm{~Hz})$ increases with dopaminergic therapy, especially during movement.

\section{References}

Alegre M, Alonso-Frech F, Rodriguez-Oroz MC, Guridi J, Zamarbide I, Valencia M, Manrique M, Obeso JA, Artieda J (2005) Movement-related changes in oscillatory activity in the human subthalamic nucleus: ipsilateral vs. contralateral movements. Eur J Neurosci 22:2315-2324.

Alexander GE, Crutcher MD (1990) Functional architecture of basal ganglia circuits: neural substrates of parallel processing. Trends Neurosci 13:266-271.

Alonso-Frech F, Zamarbide I, Alegre M, Rodriguez-Oroz MC, Guridi J, Manrique M, Valencia M, Artieda J, Obeso JA (2006) Slow oscillatory activity and levodopa-induced dyskinesias in Parkinson's disease. Brain 129:1748-1757.

Androulidakis AG, Kühn AA, Chen CC, Kempf F, Kupsch A, Schneider G-H, Doyle L, Dowsey-Limousin P, Hariz MI, Brown P (2007) Dopaminergic therapy promotes lateralised motor activity in the subthalamic area in Parkinson's disease. Brain 130:457-468.

Brown P (2003) Oscillatory nature of human basal ganglia activity: relationship to the pathophysiology of Parkinson's disease. Mov Disord 18:357-363.

Brown P, Williams D (2005) Basal ganglia local field potential activity: character and functional significance in the human. Clin Neurophysiol 116:2510-2519.

Brown P, Oliviero A, Mazzone P, Insola A, Tonali P, Di Lazzaro V (2001) Dopamine dependency of oscillations between subthalamic nucleus and pallidum in Parkinson's disease. J Neurosci 21:1033-1038.

Buzsáki G, Draguhn A (2004) Neuronal oscillations in cortical networks. Science 304:1926-1929.

Cassidy M, Brown P (2003) Spectral phase estimates in the setting of multidirectional coupling. J Neurosci Methods 127:95-103.

Cassidy M, Mazzone P, Oliviero A, Insola A, Tonali P, Di Lazzaro V, Brown P (2002) Movement-related changes in synchronization in the human basal ganglia. Brain 125:1235-1246.

Chen CC, Pogosyan A, Zrinzo LU, Tisch S, Limousin P, Ashkan K, Yousry T, Hariz MI, Brown P (2006) Intra-operative recordings of local field potentials can help localise the subthalamic nucleus in Parkinson's disease surgery. Exp Neurol 198:214-221.

Devos D, Defebvre L (2006) Effect of deep brain stimulation and L-Dopa on electrocortical rhythms related to movement in Parkinson's disease. Prog Brain Res 159:331-349.

Doyle LMF, Kühn AA, Hariz M, Kupsch A, Schneider G-H, Brown P (2005) Levodopa-induced modulation of subthalamic beta oscillations during self-paced movements in patients with Parkinson's disease. Eur J Neurosci 21:1403-1412.

Fogelson N, Pogosyan A, Kühn AA, Kupsch A, van Bruggen G, Speelman H, Tijssen M, Quartarone A, Insola A, Mazzone P, Di Lazzaro V, Limousin P, Brown P (2005) Reciprocal interactions between oscillatory activities of

different frequencies in the subthalamic region of patients with Parkinson's disease. Eur J Neurosci 22:257-266.

Fogelson N, Williams D, Tijssen M, van Bruggen G, Speelman H, Brown P (2006) Different functional loops between cerebral cortex and the subthalamic area in Parkinson's disease. Cereb Cortex 16:64-75.

Gilbertson T, Lalo E, Doyle L, Di Lazzaro V, Cioni B, Brown P (2005) Existing motor state is favored at the expense of new movement during $13-35 \mathrm{~Hz}$ oscillatory synchrony in the human corticospinal system. J Neurosci 25:7771-7779.

Hammond C, Bergman H, Brown P (2007) Pathological synchronization in Parkinson's disease: networks, models and treatments. Trends Neurosci 30:357-364.

Kaminski MJ, Blinowska KJ (1991) A new method of the description of the information flow in the brain structures. Biol Cybern 65:203-210.

Korzeniewska A, Manczak M, Kaminski M, Blinowska KJ, Kasicki S (2003) Determination of information flow direction among brain structures by a modified directed transfer function (dDTF) method. J Neurosci Methods 125:195-207.

Kühn AA, Williams D, Kupsch A, Dowsey-Limousin P, Hariz M, Schneider GH, Yarrow K, Brown P (2004) Event related beta desynchronization in human subthalamic nucleus correlates with motor performance. Brain 127:735-746.

Kühn AA, Trottenberg T, Kivi A, Kupsch A, Schneider GH, Brown P (2005) The relationship between local field potential and neuronal discharge in the subthalamic nucleus of patients with Parkinson's disease. Exp Neurol 194:212-220.

Kühn AA, Doyle L, Pogosyan A, Yarrow K, Kupsch A, Schneider GH, Hariz MI, Trottenberg T, Brown P (2006a) Modulation of beta oscillations in the subthalamic area during motor imagery in Parkinson's disease. Brain 129:695-706.

Kühn AA, Kupsch A, Schneider GH, Brown P (2006b) Reduction in subthalamic $8-35 \mathrm{~Hz}$ oscillatory activity correlates with clinical improvement in Parkinson's disease. Eur J Neurosci 23:1956-1960.

Kus R, Kaminski M, Blinowska KJ (2004) Determination of EEG activity propagation: pair-wise versus multichannel estimate. IEEE Trans Biomed Eng 51:1501-1510.

MacKinnon CD, Webb RM, Silberstein P, Tisch S, Asselman P, Limousin P, Rothwell JC (2005) Stimulation through electrodes implanted near the subthalamic nucleus activates projections to motor areas of cerebral cortex in patients with Parkinson's disease. Eur J Neurosci 21:1394-1402.

Magill PJ, Pogosyan A, Sharott A, Csicsvari J, Bolam JP, Brown P (2006) Changes in functional connectivity within the rat striatopallidal axis during global brain activation in vivo. J Neurosci 26:6318-6329.

Marsden JF, Limousin-Dowsey P, Ashby P, Pollak P, Brown P (2001) Subthalamic nucleus, sensorimotor cortex and muscle interrelationships in Parkinson's disease. Brain 124:378-388.

Maurice N, Deniau JM, Glowinski J, Thierry AM (1998) Relationships between the prefrontal cortex and the basal ganglia in the rat: physiology of the corticosubthalamic circuits. J Neurosci 18:9539-9546.

Oldfield RC (1971) The assessment and analysis of handedness: the Edinburgh inventory. Neuropsychologia 9:97-113.

Parent A, Hazrati LN (1995) Functional anatomy of the basal ganglia. I. The cortico-basal ganglia-thalamo-cortical loop. Brain Res Brain Res Rev 20:91-127.

Priori A, Foffani G, Pesenti A, Tamma F, Bianchi AM, Pellegrini M, Locatelli M, Moxon KA, Villani RM (2004) Rhythm-specific pharmacological modulation of subthalamic activity in Parkinson's disease. Exp Neurol 189:369-379.

Rappelsberger P, Petsche H (1988) Probability mapping: power and coherence analyses of cognitive processes. Brain Topogr 1:46-54.

Schlögl A, Supp GG (2006) Analyzing event-related EEG data with multivariate autoregressive parameters. Prog Brain Res 159:135-147.

Sharott A, Magill PJ, Bolam JP, Brown P (2005) Directional analysis of co- 
herent oscillatory field potentials in the cerebral cortex and basal ganglia of the rat. J Physiol (Lond) 562:951-963.

Shen B, Nadkarni M, Zappulla RA (1999) Spectral modulation of cortical connections measured by EEG coherence in humans. Clin Neurophysiol 110:115-125.

Silberstein P, Kühn A, Kupsch A, Trottenberg T, Krauss J, Wöhrle J, Mazzone P, Insola A, Di Lazzaro V, Oliviero A, Aziz T, Brown P (2003) There is a difference in patterning of globus pallidus local field potentials between PD and dystonia. Brain 126:2597-2608.

Thatcher RW, Krause PJ, Hrybyk M (1986) Cortico-cortical associations and EEG coherence: a two-compartmental model. Electroencephalogr Clin Neurophysiol 64:123-143.

Thobois S, Mertens P, Guenot M, Hermier M, Mollion H, Bouvard M, Chazot G, Broussolle E, Sindou M (2002) Subthalamic nucleus stimulation in Parkinson's disease: clinical evaluation of 18 patients. J Neurol 249:529-534.
Trottenberg T, Fogelson N, Kühn AA, Kivi A, Kupsch A, Schneider GH, Brown P (2006) Subthalamic gamma activity in patients with Parkinson's disease. Exp Neurol 200:56-65.

Trottenberg T, Kupsch A, Schneider G-H, Brown P, Kühn AA (2007) Frequency-specific distribution of local field potential activity within the subthalamic nucleus in Parkinson's disease. Exp Neurol 205:287-291.

Weinberger M, Mahant N, Hutchison WD, Lozano AM, Moro E, Hodaie M, Lang AE, Dostrovsky JO (2006) Beta oscillatory activity in the subthalamic nucleus and its relation to dopaminergic response in Parkinson's disease. J Neurophysiol 96:3248-3256.

Williams D, Tijssen M, van Bruggen G, Bosch A, Insola A, Di Lazzaro V, Mazzone P, Oliviero A, Quartarone A, Speelman H, Brown P (2002) Dopamine-dependent changes in the functional connectivity between basal ganglia and cerebral cortex in humans. Brain 125: $1558-1569$. 